\title{
Article \\ The Efficacy of Plant-Based Dietary Program in Patients with Diabetes: A Pilot Study
}

\author{
Reuben Adatorwovor ${ }^{1, *(\mathbb{D}}$, Nisha Sharma ${ }^{2}$, Dakota McCoy ${ }^{2}$, Sharon Wasserstrom ${ }^{2}$, Matthew Robinson ${ }^{2}$, \\ Jacquelyn Nyenhuis ${ }^{2}$ and Sowmya Suryanarayanan ${ }^{2}$ \\ 1 Department of Biostatistics, University of Kentucky, Lexington, KY 40536, USA \\ 2 College of Medicine, University of Central Florida, Orlando, FL 32827, USA; \\ sharman2@knights.ucf.edu (N.S.); Dakota.McCoy@knights.ucf.edu (D.M.); \\ Sharon.Wasserstrom@ucf.edu (S.W.); Matthew.Robinson@ucf.edu (M.R.); Jacque@ucf.edu (J.N.); \\ sowmya70@hotmail.com (S.S.) \\ * Correspondence: radatorwovor@uky.edu
}

Citation: Adatorwovor, R.; Sharma, N.; McCoy, D.; Wasserstrom, S.;

Robinson, M.; Nyenhuis, J.;

Suryanarayanan, $\mathrm{S}$. The Efficacy of Plant-Based Dietary Program in Patients with Diabetes: A Pilot Study. Diabetology 2021, 2, 259-271. https:// doi.org/10.3390/diabetology2040024

Academic Editor: Giancarlo Tonolo

Received: 25 October 2021

Accepted: 7 December 2021

Published: 12 December 2021

Publisher's Note: MDPI stays neutral with regard to jurisdictional claims in published maps and institutional affiliations.

Copyright: (c) 2021 by the authors. Licensee MDPI, Basel, Switzerland. This article is an open access article distributed under the terms and conditions of the Creative Commons Attribution (CC BY) license (https:// creativecommons.org/licenses/by/ $4.0 /)$.

\begin{abstract}
Dietary choices play a key role in insulin sensitivity among diabetes patients. An 8-week pilot study was conducted to evaluate whether a mostly plant-based dietary program will lead to improvement in biochemical markers in adults with diabetes. The dietary program included educational presentations, weekly cooking demonstrations and small group discussions. A sample of thirty-two adults with diabetes (types 1 and 2) were recruited and seventeen (53\%) completed the study. Matched-pair tests and Fishers exact tests were used to compare the changes in means and proportion of the participants' responses. There were changes in HbA1c, lipids, CRP (mg/L), cholesterol (mg/dL), HDL (mg/dL), triglycerides (mg/dL), LDL (mg/dL), non-HDL (mg/dL), Insulin ( $\mathrm{uIU} / \mathrm{mL})$, AST (U/L), ALT (U/L), weight (lbs), systolic blood pressure ( $\mathrm{mmHg}$ ), diastolic $(\mathrm{mmHg})$. The mean (std) age for the matched pair participant is 60.5 (11.35). Five type 1 and twelve type 2 diabetes patients showed a significant improvement in HbA1c $(p=0.001)$, weight $(p=0.002)$, intake of vegetables per day $(p$-value $=0.003)$, intake of plant-based protein $(p$-value $<0.001)$ and self-reported reduction in fatigue and improvement in energy levels. Our 8-week dietary program showed an improvement in biochemical markers and positive attitudes toward the adoption of plant-based diets.
\end{abstract}

Keywords: diabetes; HbA1c; plant-based diet; nutrition; weight

\section{Introduction}

In the United States, more than 34 million Americans ( $>1$ in 10) have been diagnosed with diabetes [1] and at least 88 million American adults (about 1 in 3) are at high risk for diabetes. Globally, the incidence of diabetes has increased to 453 million in recent years and is projected to reach 693 million by 2050. Newly diagnosed cases of type 1 and 2 diabetes have significantly increased among American youth, of which $90 \%$ are diagnosed with type 2 diabetes and 10\% with type 1 diabetes, although atypical diabetes has a mixed presentation and is also on the rise during the pandemic. COVID-19 has caused a new onset of diabetes [2], particularly type 1 diabetes and dietary measures may be potentially useful in reducing complications and improving glycemic control.

Besides exercise [3], dietary choices is a key factor driving insulin sensitivity. The consumption of low nutrient-dense foods in addition to sugar-laden beverages remain the most important etiology for developing diabetes. The landmark diabetes prevention program (DPP) trial [4] showed that dietary and lifestyle modification does not only help remit diabetes but also reverse and prevent complications. The DPP study showed a $68 \%$ reduction in the risk of developing type 2 diabetes. Plant-based diets emphasize intake of whole grains, vegetables, fruits and nuts, seeds, legumes and pulses and protein from plant sources with limited amounts of animal fats have shown potent effects in lowering 
or preventing the risk of type 2 diabetes by improvement in insulin resistance and lipid profile, in addition to other benefits.

Edge et al. [5] and Kim et al. [6] suggested that personal diet may affect the progression and development of complications in patients with type 2 diabetes. Additionally, reductions in biomarkers can result in positive outcomes for diabetic patients, as improvements in glycemic control, blood pressure and cholesterol can decrease the risk of developing diabetes-related complications [7]. A recent research review demonstrated that predominantly plant-based diets decrease all-cause mortality rates, improve glycemic control [8], markers for lipids and blood pressure and are associated with metabolic and cardiovascular benefits $[6,9]$. The authors concluded that dietary changes could be used as a preventive measure for type 2 diabetes. More importantly, they believed that this change could lead to the reduction in the use of antidiabetic medications [9].

The evidence supporting the role of glucose monitoring, exercise and medical nutrition therapy in monitoring diabetes cannot be overstated. Other forms of diabetes management include weight loss measures involving plant-based diets, pharmacotherapy and bariatric surgery. Although dietary habits and weight loss measures play key roles in diabetes management, the issue of what diet pattern best addresses hyperglycemia and its risk factors, as well as weight control, remains somewhat controversial. The idea of prolonged and uniform calorie-controlled diet plans for diabetic individuals have been replaced by individualized diabetic meal-plan approaches utilizing nutritional guidelines of the ADA and the U.S. Department of Agriculture's 2015-2020 Dietary Guidelines [10]. With this release came praise for mostly plant-based diet models, which have been extensively studied among participants with diabetes and obesity. In another study, Trapp and her colleagues [11] concluded that patronizing or adhering to mostly plant-based diets can possibly reduce the risk of developing type 2 diabetes.

A plant-based diet needs to be considered as part of diabetes patients' lifestyle changes that include the use of nutrition therapy, regular physical activity, restorative sleep, stress management, avoidance of risky substances and positive social connection as a primary therapeutic modality for treatment and reversal of chronic conditions. Adherence to this diet can be associated with fewer calories, higher nutrient density, less fat, low cholesterol, less saturated fat and, potentially, a lower risk of high BMI compared to non-followers. Indulging in healthful eating of a predominately plant-based diet has varied evidence for promoting overall health and preventing, treating and reversing diabetes [12]. Adherers often consume more fiber, potassium and vitamin rich foods including vitamin $\mathrm{C}$. This can lower their risk and prevalence for obesity, hyperglycemia and cardiovascular disease [13-15]. In support of these findings, the ADA and the American Academy of Nutrition and Dietetics [16] have now started considering mostly plant-based diets for individuals diagnosed with diabetes.

Plant-based diets have decreased levels of saturated fat, which decreases cardiovascular risk factors, and this marked decrease provides potential benefits to diabetes patients [17]. Although the benefits are intriguing, transitioning to a mostly plant-based diet can often be difficult without proper education and frequent follow-up sessions with medical professionals for type 1 and 2 diabetic patients. Many studies and conclusions have been drawn about the impact of dietary changes on type 2 diabetic patients and their health outcomes. However, no recommendations exist for mostly plant-based diets in diabetes patients, with either type 1 or 2 . Any reduction in biochemical markers could potentially result in positive outcomes for diabetic patients, as improvements in glycemic control, blood pressure and cholesterol can decrease the risk of developing diabetes-related complications [18].

One such modification in diet that has been evaluated for its effectiveness in improving biochemical markers is a low-fat mostly plant-based diet. Specifically, the study compared participants following an ADA recommended diet (15-20\% protein, $<7 \%$ saturated fat, $60-70 \%$ carbohydrates and monounsaturated fats, cholesterol $\leq 200 \mathrm{mg} /$ day) to those following a low-fat mostly plant-based diet for 22 weeks found greater improvements in $\mathrm{HbA1c}$, weight, BMI, waist circumference, total cholesterol and LDL cholesterol in the 
vegan group [19]. A low-fat, mostly plant-based diet produced a greater improvement in glycemic and plasma lipid levels as compared to the ADA dietary recommendation [20]. Other studies on plant-based diets have been shown to improve metabolic and cardiovascular health, decrease all-cause mortality rates [6], improve glycemic control and markers for lipids and blood pressure [9].

\section{Materials and Methods}

\subsection{The Aims and Objectives}

We created and implemented a dietary program with the goals of improving the life quality of these patients and transitioning them to a mostly plant-based diets. We evaluated the efficacy of our program; the efficacy of plant-based dietary programs for diabetic patients at the University of Central Florida (UCF) Health clinic. We considered two main objectives: primary and secondary aims. The primary aim included measures such as weight, blood pressure, body mass index, pulse, HbA1c, lipid profile, C-reactive protein, hepatic panel and insulin levels. These biochemical markers were chosen for their ability to signify the level of glycemic control, blood pressure and lipid profiles. The secondary endpoint involved evaluation of the participant's lifestyle and satisfaction/perceptions of the program through pre/post intervention surveys.

\subsection{Design and Materials}

This research is a longitudinal mixed methods study utilizing a survey methodology, group discussions, video lectures, large group debriefing, an educational session and cooking demonstrations. It was designed and conducted as a pilot study in the outpatient clinic setting at UCF Health and was approved by the UCF Institutional Review Board (IRB). We hypothesized that there would be no significant improvement in clinical measures including $\mathrm{HbA1c}$, weight, quality of life of the diabetes participants during the pre- and post-interventions periods.

An 8-week plant-based dietary program was created by two medical students from UCF College of Medicine with guidance from endocrinologist, nutritionist and lifestyle medicine sub-specialists. It was inspired by the following resources: Dr. Neal Barnard's Nutrition Education Curriculum Videos, 21-Day Vegan Kickstart Program, Forks over Knives documentary and App and the Plantrician Project on implementing plant-based di ets. A plant-based meal plan was given to each participant during the first week with weekly educational sessions. The educational sessions involved power point presentations that addressed the fundamentals of a plant-based diet, its benefit for the individual's health needs and how to incorporate such a plan into daily meals and activities. Some topics discussed during the educational sessions include grocery shopping tips, cooking demonstrations, ingredient alternatives, food solutions during travel and plant-based cooking recipe suggestions. In-person cooking demonstrations and food tastings were offered to allow the participants to engage in a more interactive approach to hands on cooking. This also included small group interactions where the participants encouraged each other, shared suggestions, new ingredients and cooking recipes. These sessions served as a forum for support for type 1 and 2 diabetic patients allowing patients to meet each other as well as discuss ways of overcoming some of their day-to-day struggles and share successes with each other and with study facilitators.

\subsection{Study Participants' Recruitment}

Adults 18 years and older with diabetes were primarily recruited from the Endocrinology and UCF Health clinics by in person discussion about the clinical study and design during their clinical visit. Study informational fliers were provided to help guide their decision-making process. Participants not completing both pre- and post-intervention assessments were excluded. The study promoted an adoption of a mostly plant-based diet to improve glycemic control and encourage participants to attend educational sessions on a variety of topics to enhance their understanding of the benefits of this dietary pattern. 
Out of thirty-two diabetes patients recruited, seventeen (17) completed the study, as well as both pre- and post-test survey questionnaires. The study participants included five type 1 and twelve type 2 diabetic patients. The median diabetic duration was 12 years with a mean value of 14.7 years. The initial evaluation of the participants at the baseline of the study showed that about $88 \%(n=15)$ were overweight with $47 \%(n=8)$ obese. The participants had a 2-week pre- enrollment period to get their biochemical markers and consent forms addressed and sign an informed consent, and they, they participated in an 8-week plant-based dietary program proposed and implemented to investigate the efficacy of dietary programs for diabetic patients.

\subsection{Plant-Based Dietary Intervention}

Participants in the program consented to do the following during the duration of the study:

1. Attend weekly educational sessions, cooking demonstrations and lecture presentations on the nutritional benefits of a mostly plant-based diet.

2. Keep daily dietary, blood sugar and insulin pump logs during the 8-week period.

3. Adhere to the plant-based diet during the 8-week period.

4. Complete the pre- and post-study questionnaires evaluating their overall health, nutrition, exercise and satisfaction with the plant-based dietary program during the ensuing period.

The participants were to follow the meal plan provided for the first two weeks of the study. This daily dietary plan was plant-based and included ingredients, recipes and nutritional facts for the proposed meals and snacks the participants were expected to eat. After the initial two weeks, participants had the option of either repeating the same diet plan or eating their own plant-based diet based on the nutritional education received during the weekly sessions.

The intervention was eight $2 \mathrm{~h}$ weekly sessions. Each session was run by a team of two UCF College of Medicine students, a nutritionist, endocrinologist, lifestyle medicine specialist and two medical assistants. Each session included an educational lecture 20-30 min in duration, a cooking demonstration for approximately $20 \mathrm{~min}$ followed by a $20-30 \mathrm{~min}$ group discussion session. Participants were encouraged to document their self-reported energy levels and attitudes during each week. Clinical measures were obtained through vital signs and biochemical markers as blood samples were collected at the beginning and at the end of the eight weekly sessions. These measures are reported in the results section. Since type 1 diabetic patients depend on exogenous insulin medication for glycemic control, only type 2 patients had additional insulin levels measured during lab draw.

Our secondary endpoint was participants' satisfaction and program adherence which was evaluated through questionnaires that addressed satisfaction with the program, ease of use, convenience, willingness to continue a similar plan in the future, perception on cooking and overall mood and lifestyle. We recorded pre- and post-intervention results for the 8-week dietary program. Program adherence was measured by a number of weekly sessions attended by participants, and the contents of the participants' diet logs. These endpoints: log recordings, weekly attendance and calculated percentage of plant-based meals consumed over the 8-week period for each participant were analyzed.

\subsection{Study Survey Questions and Measurement}

The pre- and post-survey questionnaires had 25 questions. There was one question on overall health of the participants, two questions on sleep, and one question on current weight status of the participants. Additional questions were on the participants' nutrition, exercise, cooking and plant-based diets. There were 13 multiple choice open response questions, two questions with yes or no responses with explanation, and the remainder were open-ended questions. The questions with ordered open responses had scales 0 to 10, with 0 representing an extreme negative connotation and 10, the most positive connotation. 
The clinical data included measurements on HbA1c, lipids, CRP (mg/L), cholesterol (mg/dL), HDL (mg/dL), triglycerides (mg/dL), LDL (mg/dL), non-HDL (mg/dL), insulin (uIU/mL), AST (U/L), ALT (U/L), weight (lbs), systolic blood pressure (mmHg) and diastolic $(\mathrm{mmHg})$ for each participant both at the baseline and at the end of the intervention period.

\section{Statistical Analysis}

Data from all study participants who responded to both pre/post intervention surveys were matched with their unique identification characteristics and were used for the statistical analysis. We analyzed responses from the participants' survey questionnaires, recorded weekly attendance and calculated the percentage of plant-based meals consumed over the 8-week period for each participant. The descriptive mean summary and the distribution of participants' demographic variables were analyzed. Open response questions were analyzed to extract the common participant responses. Descriptive analyses were performed for the survey questions, compared and hence reported. Due to the relatively small sample size, exact matched-pair tests were used to compare the differences in the mean estimates from the participants' responses to the pre/post survey matched-pair questions for each outcome variable.

The clinical measurements, dichotomized outcomes with open responses, were tested using a matched-pair t-tests. The attendance to the weekly sessions and the proportion of plant-based meals consumed over the intervention period are presented in the graphics. The efficacy of the 8-week dietary program was evaluated by testing the difference between the pre- and post-test values for various clinical markers including BMI, blood pressure and pulse.

Sensitivity analyses were conducted to assess whether the clinical marker altered the observed difference between the pre- and post-intervention measurements and between the diabetic groups. The standard 5\% statistical significance level was used for all statistical tests. R Core Team (2020) statistical software was used for all the statistical analyses.

\section{Results and Inference}

\subsection{Results}

There were seven males and 10 females enrolled in the study with a mean age of 60.5 years and standard deviation of 11.35. Tables 1-3 show the analyses of the clinical outcomes and the survey questionnaires for the 8-week intervention period. There was an improvement in the overall level of health among these participants within the 8-week period. Specifically, there was a significant improvement in clinical outcome variables: HbA1c $(p$-value $=0.001)$, weight $(p$-value $=0.002)$ and a significant lack of improvement in the systolic blood pressure $(p$-value $=0.013)$.

The survey results showed that the participants of the study had improvement in some markers during the study period. They reported better quality of life as a result of improved sleep. The study participants reported that they slept more often during the 8-week period than prior to the study. This resulted in a significant improvement $(p$-value $<0.001)$ in their energy levels during the study period. This benefit in improved sleep translates to significant improvement in weight management efforts, $p$-value $=0.041$. The nutritional benefits include a reduction in the consumption of fast food and sugary drinks, increased frequency of whole fruits, a significant $(p$-value $=0.003)$ increase in servings of vegetables per day and a significant $(p$-value $<0.001)$ increase in intake of plant-based proteins. There was a significant decrease in the amount of animal-based protein taken by the participants during this period, $p$-value $=<0.001$. Perhaps the educational sessions illuminated the potential benefits of plant-based protein over animal-based protein.

The increase in energy levels potentially led to more activities during the time of the intervention. In particular, the participants increased the number of days on which they completed moderate to strenuous intensity exercise from less than two times a week to more than three times per week during the study period. This has increased the number of 
minutes of moderate to strenuous intensity exercise from less than 30 min to more than 30 min each time, resulting in a significant improvement for this metric, $p$-value $=0.006$. Additionally, there was a significant increase $(p$-value $=0.028)$ in the number of days the participants engaged in strength/resistance training exercise per week.

The 8-week intervention was not enough to significantly change the attitude of the participants towards implementing home-based cooking. However, the intervention increased the desire to cook more meals at home which translates to increased cooking time per day and the number of meals prepared at home, as well as increased cooking for other household members including themselves, demonstrated in Figure 1. To understand the benefits of the dietary program with regard to the most salient influential factors of the dietary program, 32\% (6 out of 17) of the attendees participated because of the dietary educational sessions offered during the intervention period. Four (24\%) participants were motivated to attend the program because they desired to improve their clinical markers for their diabetes. Four (24\%) individuals also attended the program because of their willingness to manage their weight while another four participated because they wanted to learn and taste food during the cooking demonstrations. Some of the other positive factors that influenced attendance (Figure 2) of the dietary program were: to reduce the number of medications they were taking, to interact and learn strategies for managing diabetes from their peers and to reduce fatigue and improve their energy levels, highlighted in Figure 1.

Table 1. Overall measures for type 1 and 2 diabetes participants of the 8-week dietary program.

\begin{tabular}{cccc}
\hline & PRE & POST & \\
\hline Measures & Mean(SD) & Mean(SD) & 0.001 \\
\hline HbA1c & $7.3(0.74)$ & $6.8(0.57)$ & 0.720 \\
C-Reactive Protein (CRP) & $5.7(9.23)$ & $4.9(7.25)$ & 0.070 \\
Total Cholesterol & $158.6(35.31)$ & $150.1(30.79)$ & 0.093 \\
HDL & $51.5(11.12)$ & $49.2(11.12)$ & 0.378 \\
Triglycerides & $125.6(59.35)$ & $116.7(58.53)$ & 0.151 \\
LDL & $85.8(28.84)$ & $80.0(24.10)$ & 0.151 \\
Non-HDL & $107.6(30.45)$ & $100.8(26.64)$ & 0.111 \\
Insulin & $5.2(8.60)$ & $3.0(4.34)$ & 0.002 \\
BMI & $31.6(6.71)$ & $30.8(6.29)$ & 0.002 \\
Weight & $197.7(38.38)$ & $193.3(37.22)$ & 0.013 \\
Systolic Blood Pressure & $122.1(10.47)$ & $131.7(9.72)$ & 0.188 \\
Insulin Dose & $55.9(49.77)$ & $42.8(33.49)$ & 0.804 \\
Blood Sugar & $136.8(13.57)$ & $134.6(27.07)$ & 0.615 \\
AST & $19.4(5.38)$ & $21.2(7.89)$ & 0.340 \\
ALT(U/L) & $27.2(31.56)$ & $21.1(11.73)$ & \\
\hline
\end{tabular}

Table 2. Biomarkers for type 1 and 2 diabetic patients during the 8 -week dietary intervention program.

\begin{tabular}{ccccccc}
\hline & & Type II & & & Type I \\
\hline & Pre & Post & & Pre & Post \\
\hline Measures & Mean(SD) & Mean(SD) & $p$-Val & Mean(SD) & Mean(SD) & $p$-Val \\
\hline HbA1c & $7.2(0.77)$ & $6.7(0.58)$ & 0.007 & $7.6(0.58)$ & $7.2(0.33)$ & 0.089 \\
CRP & $6.7(10.83)$ & $3.8(4.57)$ & 0.352 & $3.5(3.15)$ & $7.5(11.45)$ & 0.362 \\
Total Chol & $157.3(36.50)$ & $145.8(33.05)$ & 0.061 & $162.0(36.10)$ & $160.2(24.60)$ & 0816 \\
HDL & $48.8(11.46)$ & $45.4(11.53)$ & 0.025 & $57.8(8.81)$ & $58.4(6.07)$ & 0.831 \\
Triglyceride & $148.0(56.04)$ & $137.2(57.72)$ & 0.448 & $72.0(18.81)$ & $67.6(16.50)$ & 0.610 \\
LDL & $84.8(30.15)$ & $77.3(25.98)$ & 0.137 & $88.2(28.60)$ & $86.4(19.86)$ & 0.817 \\
Non-HDL & $109.1(31.72)$ & $100.4(29.55)$ & 0.156 & $104.2(30.38)$ & $101.8(20.86)$ & 0.769 \\
Insulin & $7.4(9.49)$ & $4.3(4.63)$ & 0.111 & & - & - \\
BMI & $32.9(7.09)$ & $31.9(6.68)$ & 0.002 & $28.5(4.99)$ & $28.3(4.85)$ & 0.5553 \\
Weight & $204.6(36.02)$ & $199.0(34.99)$ & 0.002 & $181.2(42.93)$ & $179.8(4301)$ & 0.549 \\
Systolic Blood Pressure & $22.2(11.10)$ & $136.5(6.89)$ & 0.004 & $121.8(9.56)$ & $120.2(3.77)$ & 0.664 \\
Insulin Dose & $63.2(58.96)$ & $47.7(5192)$ & 0.260 & $37.5(3.53)$ & $37.9(5.99)$ & 0.656 \\
Blood Sugar & $137.9(11.75)$ & $126.33(23.91)$ & 0.246 & $134.3(18.86)$ & $153.3(27.17)$ & 0.276 \\
AST & $18.7(5.21)$ & $21.4(7.75)$ & 0.509 & $20.8(6.06)$ & $20.6(9.12)$ & 0.938 \\
ALT(U/L) & $30.58(37.09)$ & $21.6(9.42)$ & 0.323 & $19.0(11.55)$ & $19.8(17.4)$ & 0.789 \\
\hline
\end{tabular}


Table 3. Analysis of the mean difference between the pre- and post-test survey questionnaires.

\begin{tabular}{lcc}
\hline \multicolumn{1}{c}{ Questions } & Difference & $p$-Value \\
\hline Overall level of health & 0.346 & 0.230 \\
Average sleep in a 24-h & -0.033 & 0.818 \\
Feel tired or difficulty staying awake & -0.600 & $<0.001$ \\
Weight management efforts & -0.267 & 0.041 \\
Frequency of fast food and sugary drinks & -0.346 & 0.121 \\
Frequency of whole fruits servings & 0.179 & 0.239 \\
Servings of vegetables per day & 0.714 & 0.003 \\
Frequency of animal-based proteins (chicken, turkey) & -1.642 & 0.001 \\
Frequency of plant-based proteins (beans, lentils) & 1.692 & $<0.001$ \\
Days of moderate to strenuous intensity exercise & 0.267 & 0.301 \\
Minutes of moderate to strenuous intensity exercise & -0.533 & 0.006 \\
Days of strength/resistance training exercise per week & 0.400 & 0.028 \\
Cooking meals & 0.067 & 0.334 \\
Time spent cooking in a day & 0.167 & 0.504 \\
Cooked meals in a day & 0.250 & 0.275 \\
Number of cooked meals for other people & 0.071 & 0.690 \\
Enjoy cooking & 0.583 & 0.170 \\
Cook for myself & -0.083 & 0.339 \\
Difficulty associated with transition to plant-based diet & 1.358 & 0.007 \\
Willingness to permanently adopt a plant-based diet & -0.267 & 0.383 \\
Willingness to continue with diet/exercise programs & 0.200 & 0.510 \\
\hline
\end{tabular}

\section{Positive Factors of the Plant-Based Dietary Program}

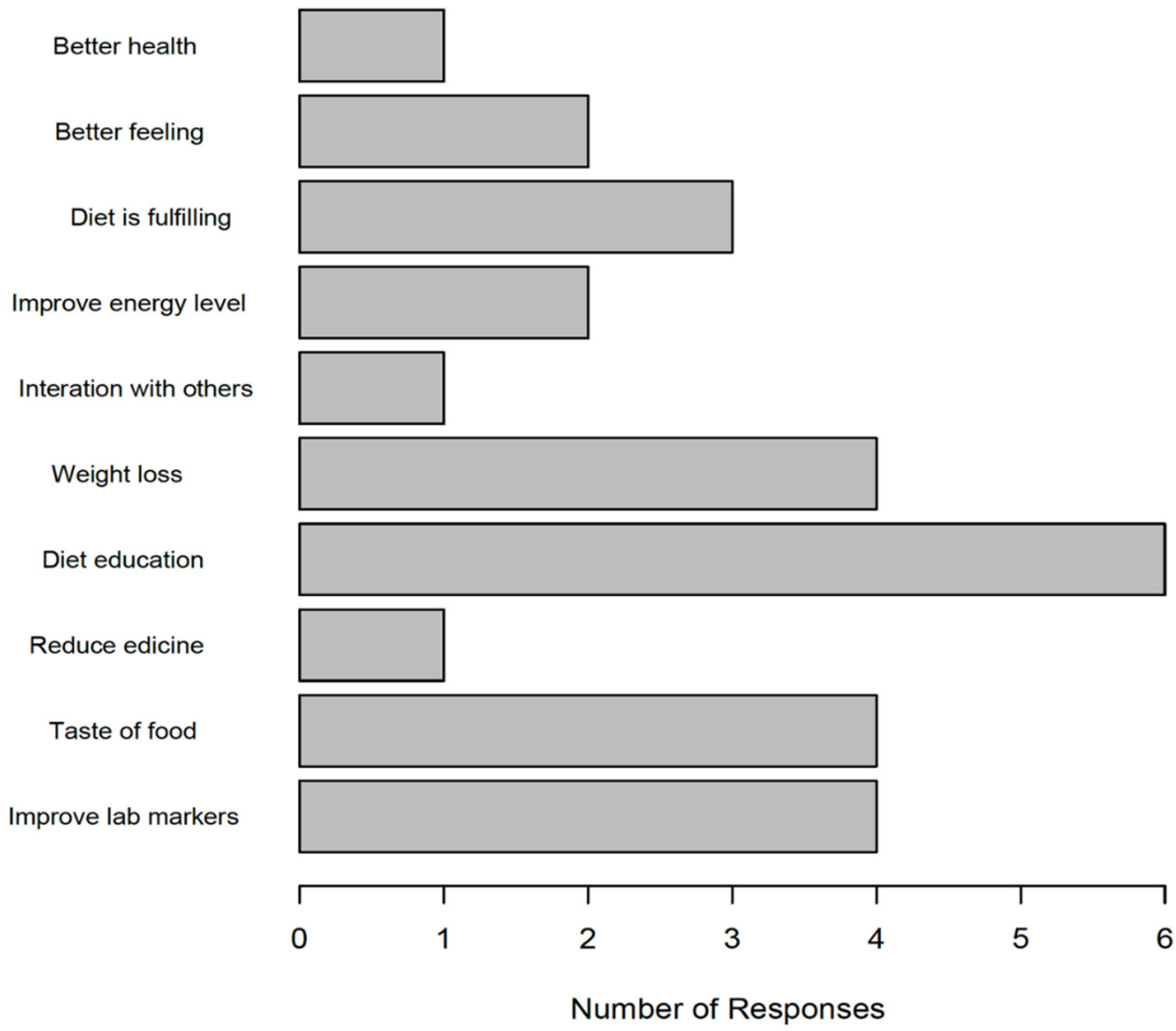

Figure 1. The Desirable Elements of the Plant-Based Dietary Program. 


\section{Attendance for Weekly Educational Session and Cooking Demonstration}

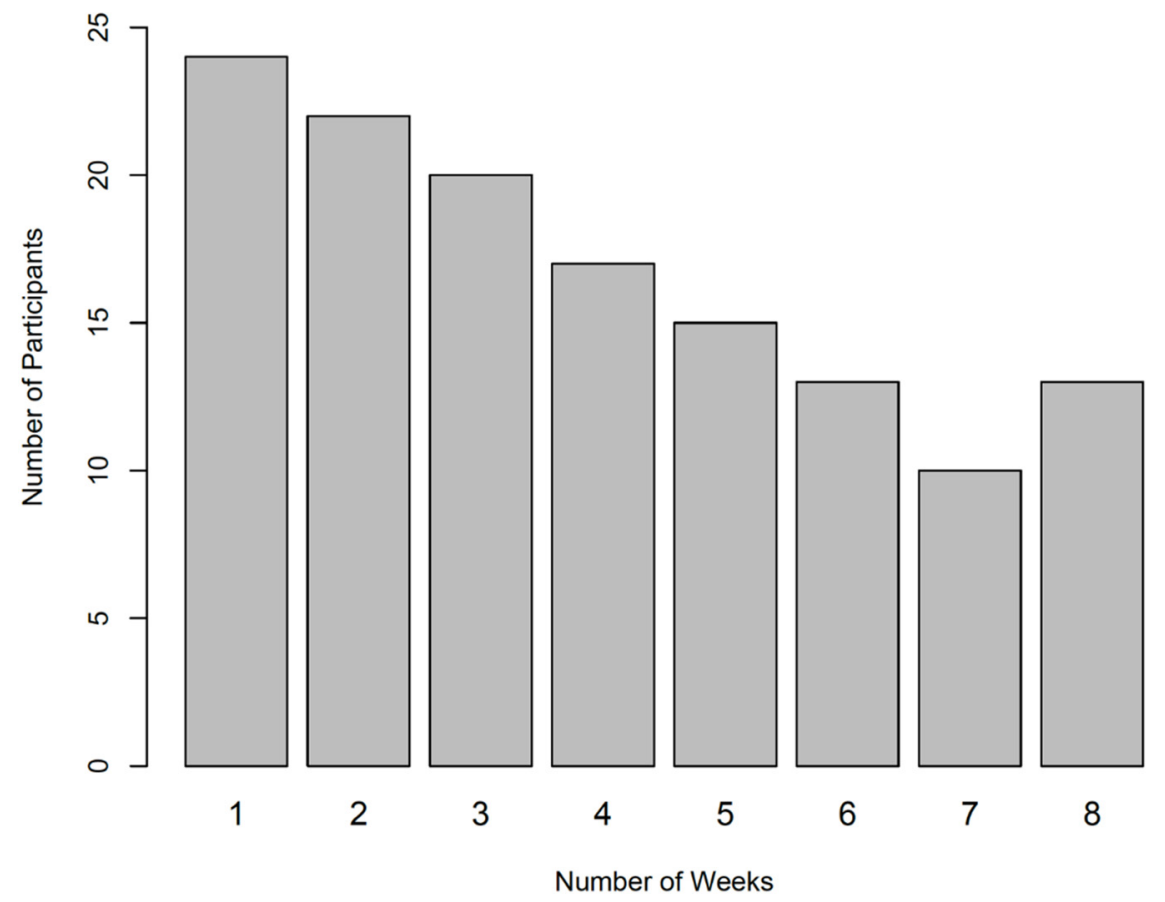

Figure 2. Weekly Attendance Sessions.

Participants of the study showed an increased desire for the plant-based dietary program. There was a significant increase in attitudes associated with the implementation of a plant-based diet among the study participants ( $p$-value $=0.007$ ). Most of them believed that it was relatively easy to transition to plant-based diets once introduced to it. This was evident in their willingness to permanently adopt and maintain plant-based diets (Figure 3) and their desire to continue with dietary and exercise programs instituted by their healthcare providers.

Table 2 shows the comparison of clinical measures for type 1 and 2 diabetic patients. The results of the primary endpoint show a significant decrease in $\mathrm{HbA1c}$, and HDL for type 2 diabetes patients with $p$-values 0.007 and 0.025 , respectively. Although we observe changes in values from the pre- to post-intervention period for type 1 diabetes patients, it was not statistically significant. There were decreases in endogenous insulin production during the intervention period.

\subsection{Inference}

During the 8-week period, there was a marked reduction in weight and the mean $\mathrm{HbA1c}$ values comparing pre- to post-intervention measures, a reduction in total cholesterol indicating reduced risks during this period, but a decrease in HDL cholesterol. The observed decrease in the mean HDL levels during the study period might be due to the low fat-diet [21] characterized by a high intake of fruit and vegetables, or due to lack of exercise, although we do not know the association of cardiovascular protection or reduction therein due to low HDL levels. We observed a reduction in intakes of triglycerides and an elevated level of the systolic blood pressure during this period. A plausible explanation for this might be due to increased stress among the participants prior to their post-intervention measurements due to it being the last day of the program. There was a slight decrease in the overall dose of insulin medication and corresponding blood sugar levels during the intervention period and an overall improvement of $\mathrm{HbA} 1 \mathrm{c}$. 


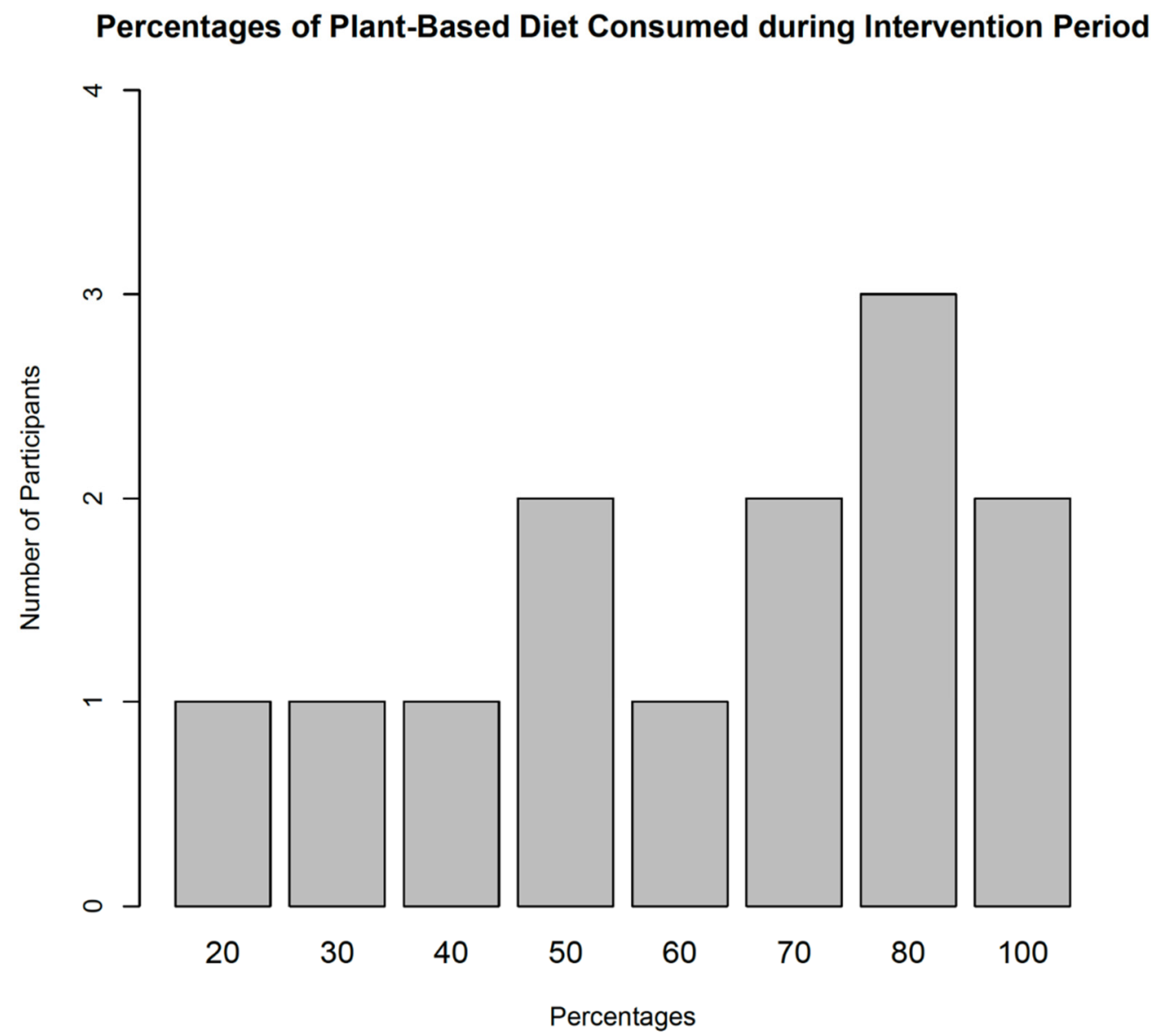

Figure 3. Proportion of Meal Intakes.

\section{Discussion}

\subsection{Findings}

Our findings from this pilot program confer $\mathrm{HbA} 1 \mathrm{c}$ lowering as noted in other studies [22]. Mostly plant-based diets can help improve the outcomes among individuals diagnosed with diabetes. We investigated the effect of a mostly plant-based dietary program on individuals living with diabetes. Participants reported feeling less tired. This suggests that a mostly plant-based diet is beneficial to diabetic patients due to improvements in their clinical markers and their overall health. HDL levels were lower at the end of the intervention which confirms what has been shown in other studies [23]. However, the mechanism for lower HDL remains unclear as many of these diets have been thought to be beneficial to people with cardiovascular risk. The possible mechanism is believed to lower Apo A-1 production rates, an increase physical activity or possibly overall reductions in all lipid subfractions.

A major theme observed during the intervention sessions was that the plant- based dietary program was easy to implement both at home and when travelling. In effect, it is hoped that the adoption of a mostly plant-based diet will potentially reduce the number of direct contacts with the health-care system thereby reducing the strain of higher demand on healthcare professionals. We observed improved estimates for the markers, but this was not significant among the type 1 diabetic patients and a large-scale study might be needed to evaluate the benefits in such a subgroup. This might be effective also in group settings with multidisciplinary teams including endocrinology, lifestyle medicine and nutrition to evaluate them on a joint basis and help with the motivation to adhere to and plan these diets.

The 8-week plant-based diet program was a tool that enhanced the understanding of the benefits associated with transitioning to a mostly plant-based dietary program. An 
effective plant-based diet program may incorporate regular meetings and educational sessions while also considering the participants' financial status, time availability, meal preparation and gastrointestinal sensitivity. The dietary program not only improved the participants' understanding of the benefits of plant-based diets but also offered an alternative way of managing chronic diseases.

\subsection{Study Limitation}

The major limitations of this pilot study were the small sample size for both type 1 and 2 diabetic patients, and the short study duration with no control arm. These patients were mainly from the UCF Health Clinic which could potentially impact the generalization of our findings. Subsequent studies should sample from other hospitals and clinics thereby increasing the sample size, and potentially over-sample type 1 patients. This will enable researchers to tease out any difference that may exist in biochemical markers like HDL-C, LDL-C or triglyceride levels across time. With a large sample size, a study evaluating the benefits in type 1 diabetes patients would help in-depth evaluation with continuous glucose monitoring of glycemic patterns effectively focusing on glycemic variability which are associated with the highest risk of index complications in diabetes patients, particularly microvascular and macrovascular complications in the long run. We did not evaluate the effect of genetic predisposition and metabolism of participant's health.

Current dietary intakes of participants should be documented prior to the start of the intervention program to account for any potential effects of dietary behaviors on the outcome. For example, a couple of participants had begun transitioning to a vegetarian diet prior to the start of our intervention program. Dietary logs (Figure 3) and questionnaires should be digitized to facilitate accessibility and dietary intakes monitoring and help ease transcription of data.

\subsection{Remark}

Vegetarian and mostly plant-based diets have been shown in observational studies to have some benefits in diabetes management, with participants less likely to develop diabetes as compared to those following non-plant-based diets [20,23]. Vegetarian diets are low in fat and higher in carbohydrates and may lead to changes in lipid levels. Our study also confirmed the findings reported in observational studies and some type 2 diabetes investigational trials in that there was an improvement in $\mathrm{HbA} 1 \mathrm{c}$ levels and a decrease in HDL levels and weight loss that was statistically significant. We observed lower levels of lipids, lower total cholesterol, LDL and HDL levels, although triglyceride levels can be higher ${ }^{15}$. In some studies, higher glycemic index foods caused an increase in LDL-C which was concerning $[15,20]$.

Overall, the benefit of an 8-week mostly plant-based diet in diabetic patients was mainly seen in weight loss and reduction in intramyocellular lipid concentrations. This might explain the situation wherein, although not well understood, the decrease in HDL levels is not actually associated with higher cardiac risk as shown in the article by Kent et al. [24] which even goes on to question the usefulness of measuring HDL-C in vegetarian diets. Possible mechanisms for explaining lower HDL-C based on Barnard et al. [20] was lower Apo A- 1 production. However, another contributing factor which is lower total cholesterol levels in general with improvement of triglyceride and LDL-C levels and overall decrease in HDL-C would be a possibility if a truly low fat, low glycemic index vegetarian or vegan diet is chosen.

Our study confirmed that HDL-C decreased with our plant-based dietary program. Although there was improvement in other markers of lipid profile and inflammation, these estimates were not statistically significant given the smaller sample size for our pilot study. Mostly plant-based diets can help improve markers in type 2 diabetes patients but there has not been the same type of observational studies or investigational trials in type 1 diabetes, and this pilot project hopefully will create the interest to study populations with type 1 diabetes patients. Our study aims to mainly help create interest in bringing larger 
studies to evaluate the beneficial effects of plant-based diets. Analysis of the questionnaires show that by the end of the program, most patients believed that transitioning to a mostly plant-based program is not as difficult as initially perceived. This may be due to the social aspect of participating in a plant-based diet which may help individuals in the transition into the plant-based diet. The questionnaires also show that the most helpful part of transitioning into a plant-based diet was attending the weekly sessions and understanding the health benefits of a plant-based diet. This can reflect the benefits of including weekly sessions when planning to implement a successful diet transition program. Throughout the study, participants quoted that they enjoyed meeting other individuals who were also in the same situation as they partook in the plant-based diet program. The most difficult part of transitioning into the diet was preparing the meals. This is a major issue in our society where eating a plant-based meal can sometimes be more expensive than an animal-based meal.

\section{Conclusions}

The 8-week dietary program interventions demonstrated an increased association between plant-based diets and improvements in physical and psychological well-being, as well as improvements in $\mathrm{HbA1c}$ control [8] in individuals diagnosed with diabetes. Additionally, the educational sessions and cooking demonstrations helped improve the understanding of the benefits of mostly plant-based diets in the management of diabetes. These improvements translated to a discontinuation or reduction in the use of diabetesrelated medications such as insulin doses among participants post-intervention results. These results were similar to the findings of Toumpanakis et al. [25]. Our study supports the recommendations of the Centers for Disease Control and Prevention guidelines for diabetes and prediabetes control [26].

Data for changes in $\mathrm{HbA1c}$ and weight were statistically significant with $p$-values 0.001 and 0.002 , respectively, shown in Table 1 , suggesting this plant-based diet program was effective in helping diabetes mellitus (DM) patients improve their glycemic control (Table 3). These improvements in biochemical markers could potentially result in better health outcomes. HDL change, despite it decreasing, is a common finding reported in some mostly plant-based diet studies. The real reason behind this is unknown, although possible causes are lowering of Apo A levels or overall improvement in cholesterol levels, hence lowering HDL levels. A statistically significant decrease in $\mathrm{HbA1c}$ was not found for type $1 \mathrm{DM}$ patients when analyzed alone $(p=0.089)$. Lack of significant change in biochemical markers in type 1 diabetes patients may be due to the small sample size. However, the program may have some clinical significance, demonstrating levels moving towards ADA recommendations (Table 1; Table 3).

The questionnaire responses showed that the mostly plant-based diet program had a positive impact on diabetic patients. Participants reported lower fatigue levels and increased weekly exercise. The majority of the participants rated the effectiveness of the program as $5 / 5$. Considering these results from the primary and secondary endpoints, we conclude that this plant-based dietary program may be beneficial to all diabetic patients of UCF Health and consideration should be made towards group diabetes sessions which would benefit all interested in making healthier choices with diet. Furthermore, the possibility of having larger scale trials looking at type 1 diabetes subjects and benefits as noted with type 2 diabetes subjects as well. This also brings to light the fact that well planned vegetarian and vegan diets rich in fiber and low in glycemic index and fat can have far ranging benefits which would help revise ADA guidelines in terms of recommending such diets to all diabetic populations.

Author Contributions: Conceptualization, S.W.; methodology, S.W., S.S., N.S., D.M., J.N., M.R., R.A.; software, R.A.; formal analysis, M.R., R.A.; investigation, S.S., N.S., D.M.; data curation, N.S., D.M., S.W.; writing—original draft preparation, R.A.; writing—review and editing, R.A., S.W., S.S., N.S., D.M., J.N., M.R.; visualization, R.A., M.R.; supervision and project administration, S.S. and S.W. and J.N. All authors have read and agreed to the published version of the manuscript. 
Funding: None declared.

Institutional Review Board Statement: The study was conducted according to the guidelines of the Declaration of Helsinki, and approved by the Institutional Review Board University of Central Florida with protocol number STUDY00000507 and approved on 8 October 2019.

Informed Consent Statement: Informed consent was obtained from all subjects involved in the study.

Data Availability Statement: The data for the analysis can be found on GitHub at https:/ / github. com/reubenadat/MDPI/blob/f5175b3bb57deb89175c62b0be72e5025d939303/PlantBased.xlsx.

Acknowledgments: To Kera Pederson and Barbara Naron-nurses, front office staff at UCF health Clinic administration, helped provide a large conference room to facilitate participants check-in, video and live demonstrations.

Conflicts of Interest: The authors declare that there is no conflict of interest regarding the publication of this article.

\section{References}

1. Centers for Disease Control and Prevention; US Department of Health and Human Services. National Diabetes Statistics Report, 2020; Centers for Disease Control and Prevention, US Department of Health and Human Services: Atlanta, GA, USA, 2020.

2. Rubino, F.; Amiel, S.A.; Zimmet, P.; Alberti, G.; Bornstein, S.; Eckel, R.H.; Mingrone, G.; Boehm, B.; Cooper, M.E.; Chai, Z.; et al. New-Onset Diabetes in Covid-19. N. Engl. J. Med. 2020, 383, 789-790. [CrossRef]

3. Colberg, S.R.; Sigal, R.J.; Fernhall, B.; Regensteiner, J.G.; Blissmer, B.J.; Rubin, R.R.; Chasan-Taber, L.; Albright, A.L.; Braun, B. Exercise and type 2 diabetes: The American College of Sports Medicine and the American Diabetes Association: Joint position statement. Diabetes Care 2010, 33, e147-e167. [CrossRef] [PubMed]

4. Group DPPDR. The Diabetes Prevention Program (DPP): Description of lifestyle intervention. Diabetes Care 2002, $25,2165-2171$. [CrossRef] [PubMed]

5. Edge, J.A.; Ford-Adams, M.E.; Dunger, D.B. Causes of death in children with insulin dependent diabetes 1990-1996. Arch. Dis. Child. 1999, 81, 318-323. [CrossRef] [PubMed]

6. Kim, H.; Caulfield, L.E.; Garcia-Larsen, V.; Steffen, L.M.; Coresh, J.; Rebholz, C.M. Plant-Based Diets Are Associated With a Lower Risk of Incident Cardiovascular Disease, Cardiovascular Disease Mortality, and All-Cause Mortality in a General Population of Middle-Aged Adults. J. Am. Heart Assoc. 2019, 8, e012865. [CrossRef] [PubMed]

7. Brach, J.S.; Talkowski, J.B.; Strotmeyer, E.; Newman, A.B. Diabetes Mellitus and Gait Dysfunction: Possible Explanatory Factors. Phys. Ther. 2008, 88, 1365-1374. [CrossRef] [PubMed]

8. Dunaief, D.M.; Fuhrman, J.; Dunaief, J.L.; Ying, G. Glycemic and cardiovascular parameters improved in type 2 diabetes with the high nutrient density (HND) diet. Open J. Prev. Med. 2012, 2, 364-371. [CrossRef]

9. Kahleova, H.; Levin, S.; Barnard, N. Cardio-metabolic benefits of plant-based diets. Nutrients 2017, 9, 848. [CrossRef] [PubMed]

10. You, A. Dietary Guidelines for Americans; US Department of Health and Human Services and US Department of Agriculture: New York, NY, USA, 2015.

11. Trapp, C.; Levin, S. Preparing to Prescribe Plant-Based Diets for Diabetes Prevention and Treatment. Diabetes Spectr. 2012, 25, 38-44. [CrossRef]

12. Trapp, C.B.; Barnard, N.D. Usefulness of Vegetarian and Vegan Diets for Treating Type 2 Diabetes. Curr. Diabetes Rep. 2010, 10, 152-158. [CrossRef]

13. Berkow, S.E.; Barnard, N. Vegetarian diets and weight status. Nutr. Rev. 2006, 64, 175-188. [CrossRef] [PubMed]

14. Appleby, P.N.; Davey, G.K.; Key, T.J. Hypertension and blood pressure among meat eaters, fish eaters, vegetarians and vegans in EPIC-Oxford. Public Health Nutr. 2002, 5, 645-654. [CrossRef] [PubMed]

15. Ferdowsian, H.R.; Barnard, N.D. Effects of plant-based diets on plasma lipids. Am. J. Cardiol. 2009, 104, 947-956. [CrossRef] [PubMed]

16. American Diabetes Association. Standards of medical care in diabetes-2014. Diabetes Care 2014, 37, S14. [CrossRef]

17. Yu, C.; Chen, Y.; Cline, G.W.; Zhang, D.; Zong, H.; Wang, Y.; Bergeron, R.; Kim, J.K.; Cushman, S.W.; Cooney, G.J.; et al. Mechanism by which fatty acids inhibit insulin activation of insulin receptor substrate-1 (IRS-1)-associated phosphatidylinositol. J. Biol. Chem. 2002, 277, 50230-50236. [CrossRef] [PubMed]

18. Deshpande, A.D.; Harris-Hayes, M.; Schootman, M. Epidemiology of diabetes and diabetes-related complications. Phys. Ther. 2008, 88, 1254-1264. [CrossRef] [PubMed]

19. Barnard, N.D.; Cohen, J.; Jenkins, D.J.; Turner-McGrievy, G.; Gloede, L.; Jaster, B.; Seidl, K.; Green, A.A.; Talpers, S. A Low-Fat Vegan Diet Improves Glycemic Control and Cardiovascular Risk Factors in a Randomized Clinical Trial in Individuals with Type 2 Diabetes. Diabetes Care 2006, 29, 1777-1783. [CrossRef] 
20. Barnard, N.D.; Cohen, J.; Jenkins, D.J.A.; Turner-McGrievy, G.; Gloede, L.; Green, A.; Ferdowsian, H. A low-fat vegan diet and a conventional diabetes diet in the treatment of type 2 diabetes: A randomized, controlled, 74-wk clinical trial. Am. J. Clin. Nutr. 2009, 89, 1588S-1596S. [CrossRef]

21. Brinton, E.A.; Eisenberg, S.; Breslow, J.L. A low-fat diet decreases high density lipoprotein (HDL) cholesterol levels by decreasing HDL apolipoprotein transport rates. J. Clin. Investig. 1990, 85, 144-151. [CrossRef]

22. McMacken, M.; Shah, S. A plant-based diet for the prevention and treatment of type 2 diabetes. J. Geriatr. Cardiol. 2017, 14, 342-354. [CrossRef] [PubMed]

23. Yokoyama, Y.; Levin, S.M.; Barnard, N.D. Association between plant-based diets and plasma lipids: A systematic review and meta-analysis. Nutr. Rev. 2017, 75, 683-698. [CrossRef] [PubMed]

24. Kent, L.; Morton, D.; Rankin, P.; Ward, E.; Grant, R.; Gobble, J.; Diehl, H. The effect of a low-fat, plant-based lifestyle intervention (CHIP) on serum HDL levels and the implications for metabolic syndrome status-a cohort study. Nutr. Metab. 2013, 10, 1-6. [CrossRef] [PubMed]

25. Toumpanakis, A.; Turnbull, T.; Alba-Barba, I. Effectiveness of plant-based diets in promoting well-being in the management of type 2 diabetes: A systematic review. BMJ Open Diabetes Res. Care 2018, 6, e000534. [CrossRef] [PubMed]

26. Centers for Disease Control and Prevention; US Department of Health and Human Services. National Diabetes Statistics Report, 2017; Centers for Disease Control and Prevention, US Department of Health and Human Services: Atlanta, GA, USA, 2017. 